\title{
Effective potential and spontaneous symmetry breaking in the noncommutative $\varphi^{6}$ model
}

\author{
G. D. Barbose* \\ Centro Brasileiro de Pesquisas Físicas, CBPF, \\ Rua Dr. Xavier Sigaud 150, 22290-180, Rio de Janeiro, Brazil
}

\begin{abstract}
We study the conditions for spontaneous symmetry breaking of the $(2+1)$-dimensional noncommutative $\varphi^{6}$ model in the small- $\theta$ limit. In this regime, considering the model as a cutoff theory, it is reasonable to assume translational invariance as a property of the vacuum state and study the conditions for spontaneous symmetry breaking by an effective potential analysis. An investigation of up to the two loop level reveals that noncommutative effects can modify drastically the shape of the effective potential. Under reasonable conditions, the nonplanar sector of the theory can become dominant and induce symmetry breaking for values of the mass and coupling constants not reached by the commutative counterpart.

PACS numbers: 11.10.Nx,11.10.Lm,12.38.Bx
\end{abstract}

\section{INTRODUCTION}

Quantum field theories with canonical noncommutativity of particle coordinates became a subject of intensive investigation over the past years [1, 2]. Characterized by the relation

$$
\left[\hat{x}^{\mu}, \hat{x}^{\nu}\right]=i \theta^{\mu \nu},
$$

where $\theta^{\mu \nu}$ is an antisymmetric constant tensor, these theories were shown to emerge as a natural approximation of string theory in its low-energy limit [3], in D-brane physics and matrix theory [4], and in the context of semiclassical gravity [5]. They have also proved to be applicable to condensed matter physics for the description of the quantum Hall effect [6] and superconductivity [7].

Among many interesting phenomena discovered in the study of noncommutative quantum field theories, we quote Lorentz violation [8], nonlocality [2], and IR-UV mixing [9]. The latter was shown to have deep consequences, such as the modification of the conventional Wilsonian picture of renormalization group flows in the very-low-momentum domain [2, 10] and a change in the phase structure of a number of models [11]. From the experimental point of view, several attempts to establish an empirical basis for noncommutativity are also under consideration (see, e.g., 12, 13]).

Investigations were carried out to understand the role of noncommutativity in the process of spontaneous symmetry breaking (SSB), most of them focused on the $\varphi^{4}$ model 10, 11, 14, 15, 16, 17]. Currently, there are few investigations in (2+1)-dimensional $\varphi^{6}$ theory [18, 19]. Reference 19] is the only one on SSB, where an $O(N)$ model is analyzed with emphasis on the issue of IR-UV mixing and renormalizability aspects.

The aim of the present work is to verify how noncommutativity may affect the behavior of the $\varphi^{6}$ model as long as the conditions for SSB are considered. We shall work with a cutoff field theory. Our motivation for this comes mainly from three reasons. Until now, it has been unknown if the noncommutative $\varphi^{6}$ model is renormalizable. The renormalizability of noncommutative field theories is still under discussion (see, e.g., 11, 14, 17, 20, 21] and references therein). The second motivation is that, recently, numerical simulations [22] are corroborating results provided by noncommutative field theories based on a Wilsonian approach [1], where a cutoff momentum is introduced. Therefore, the cutoff models can provide interesting information on the vacuum structure of field theories. A third reason comes from the fact that, after all, quantum field theory is presently in the era of

*Electronic address: gbarbosa@cbpf.br 
effective field theories [23]. The most successful theories, which are renormalizable, are nowadays understood as low-energy approximations of a more fundamental theory (perhaps not yet a field theory) and thus have their validity expected to be limited up to an energy scale. Additional motivation, from the phenomenological point of view, to consider the noncommutative models as cutoff theories may be found in [13].

We shall restrict our considerations to the case of small $\theta$ and assume translational symmetry as a property of the vacuum state. This assumption was recently reassessed in the framework of noncommutative field theory (see, e.g., 11, 15]). The argument behind these papers is that, as a consequence of noncommutativity, the vacuum state of the models is no longer realized for a constant $\varphi_{c l}(x)$. When this is the case, the effective potential is not a useful tool to analyze SSB. However, as pointed out in [11], the particular case where $\theta$ is small, SSB is expected to occur from a uniform-ordered to a disordered domain. Actually, this prediction seems to be connected to the adoption of an explicit cutoff for the theory, as we shall discuss later. It is interesting to quote that the existence of a uniform-ordered domain was verified in a nonperturbative calculation 22 involving the three-dimensional $\varphi^{4}$ model. Once a system is found in such a state, the minimum of the effective action is realized for a constant $\varphi_{c l}$ and translational symmetry is a property of the vacuum state.

The noncommutative effects relevant in the regime under consideration come from the crossing of internal lines in the Feynman diagrams. Since the finiteness of some of these diagrams is attributed to an effective cutoff [9] $\Lambda_{\text {eff }} \sim 1 / \sqrt{\theta}$, introduced by noncommutativity, for $\theta$ sufficiently small these diagrams may become dominant and drastically modify the behavior of the field theories. Indeed, this is exactly what will be shown to occur in this work.

This paper is organized as follows. Section II presents the noncommutative version of the $\varphi^{6}$ model and path integral method employed for the perturbative calculation of the effective potential. In Sec. III, the effective potential is evaluated up to two-loop order. Section IV is devoted to an analysis of the properties of the effective potential and the conditions for SSB. Finally, in Sec. V, we end up with a general discussion and outlook.

\section{NONCOMMUTATIVE $\varphi^{6}$ MODEL}

\section{A. Background}

One important aspect concerning Eq. (1) is that the transformation properties of the indices $\mu$ and $\nu$ is not the same on the left- and right-hand sides. The two sides carry Lorentz indices and transform under coordinate changes, which characterize the observer (frame) Lorentz transformations. However, the right-hand side is invariant under particle Lorentz transformations, which do not act on $\theta^{\mu \nu}$ [8]. This explicitly shows how particle Lorentz symmetry is broken by noncommutativity. Since $\theta^{\mu \nu}$ is Lorentz-observer covariant, we can find a rigid orthogonal frame transformation $\tilde{x}=L x$ that takes the $\theta^{\mu \nu}$-matrix to its off-diagonal block form:

$$
L\left[\begin{array}{ccc}
0 & \theta^{01} & \theta^{02} \\
-\theta^{01} & 0 & \theta^{12} \\
-\theta^{02} & -\theta^{12} & 0
\end{array}\right] L^{T}=\left[\begin{array}{ccc}
0 & 0 & 0 \\
0 & 0 & \theta \\
0 & -\theta & 0
\end{array}\right],
$$

where $\theta^{2}=\left(\theta^{01}\right)^{2}+\left(\theta^{02}\right)^{2}+\left(\theta^{12}\right)^{2}$. Thus, in an intrinsic three-dimensional world, with the appropriate choice for the physical frame, noncommutativity of the space and time coordinates can be reduced to a pure spatial noncommutativity, characterized by a unique parameter $\theta$.

Working with a noncommutative quantum field theory is equivalent to working with a commutative quantum field theory by replacing the usual product in the action by the star product of functions. The latter is defined as

$$
(f \star g)(x)=\left.\exp \left(\frac{i}{2} \theta^{\mu \nu} \frac{\partial}{\partial \xi^{\mu}} \frac{\partial}{\partial \eta^{\nu}}\right) f(x+\xi) g(x+\eta)\right|_{\xi=\eta=0,}
$$


and satisfies

$$
\begin{aligned}
\int d^{3} x \phi_{1}(x) \star \phi_{2}(x) \ldots \phi_{n}(x) & =\int \frac{d^{3} k_{1}}{(2 \pi)^{3}} \ldots \frac{d^{3} k_{n}}{(2 \pi)^{3}}(2 \pi)^{3} \delta^{3}\left(k_{1}+\ldots+k_{n}\right) \\
& \times \exp \left(-\frac{i}{2} \sum_{i<j} k_{i \mu} \theta^{\mu \nu} k_{j \nu}\right) \phi_{1}\left(k_{1}\right) \phi_{2}\left(k_{2}\right) \ldots \phi_{n}\left(k_{n}\right) .
\end{aligned}
$$

\section{B. Formulation}

For the evaluation of the effective potential, we shall use path integral methods 24, 25. The Euclidean action of the noncommutative $\varphi^{6}$ theory is written as

$$
S=\int \frac{d^{3} x}{(2 \pi)^{3}}\left[\frac{1}{2} \partial^{\mu} \tilde{\varphi} \partial_{\mu} \tilde{\varphi}+\frac{1}{2} m^{2} \tilde{\varphi}^{2}+\frac{g}{4 !} \tilde{\varphi} \star \tilde{\varphi} \star \tilde{\varphi} \star \tilde{\varphi}+\frac{f}{6 !} \tilde{\varphi} \star \tilde{\varphi} \star \tilde{\varphi} \star \tilde{\varphi} \star \tilde{\varphi} \star \tilde{\varphi}\right] .
$$

Spontaneous symmetry breaking is introduced by allowing the quantum field, $\tilde{\varphi}$ to acquire a nonvanishing vacuum expectation value,

$$
\varphi_{c l}(x)=\langle 0|\tilde{\varphi}(x)| 0\rangle_{J},
$$

while the effective action $\Gamma\left[\varphi_{c l}\right]$ develops an absolute minimum for $\varphi_{c l}(x)=\langle 0|\tilde{\varphi}(x)| 0\rangle_{J=0}$.

The perturbative loopwise expansion for the computation of $\Gamma\left[\varphi_{c l}\right]$ via quantum corrections to Eq. (5) must be performed around a stable vacuum. In order to do this, we substitute $\tilde{\varphi}=\varphi_{c l}+\varphi$ in Eq. (5) and expand around $\varphi_{c l}$. This yields

$$
\begin{aligned}
\mathcal{L} & =\frac{1}{2} \partial_{\mu} \varphi \partial^{\mu} \varphi+\frac{1}{2}\left(m^{2}+\frac{1}{2} g \varphi_{c l}^{2}+\frac{1}{24} f \varphi_{c l}^{4}\right) \varphi^{2}+\frac{1}{3 !}\left(g \varphi_{c l}+\frac{1}{6} f \varphi_{c l}^{3}\right) \varphi \star \varphi \star \varphi \\
& +\frac{1}{4 !}\left(g+\frac{1}{2} f \varphi_{c l}^{2}\right) \varphi \star \varphi \star \varphi \star \varphi+\frac{1}{5 !}\left(f \varphi_{c l}\right) \varphi \star \varphi \star \varphi \star \varphi \star \varphi+\frac{1}{6 !} f \varphi \star \varphi \star \varphi \star \varphi \star \varphi \star \varphi
\end{aligned}
$$

where the linear term in the fields disappears for its coefficient is the classical field equation. To simplify the manipulations, we shall use the notation

$$
M^{2}=m^{2}+\frac{1}{2} g \varphi_{c l}^{2}+\frac{1}{24} f \varphi_{c l}^{4}, \quad A=g \varphi_{c l}+\frac{1}{6} f \varphi_{c l}^{3}, \quad B=g+\frac{1}{2} f \varphi_{c l}^{2}, \quad C=f \varphi_{c l} .
$$

The Euclidean effective action is given, in the functional formalism, by the expression 24, 25.

$$
\Gamma\left[\varphi_{c l}\right]=\int d^{3} x \mathcal{L}\left(\varphi_{c l}\right)+\frac{\hbar}{2} \ln \operatorname{det}\left(\frac{\partial^{2} \mathcal{L}}{\partial \varphi \partial \varphi}\right)-(\text { connected } 1 \text { PI diagrams }) .
$$

As far as one assumes translational symmetry as a property of the model, the vacuum structure of the theory can be determined by studying $\Gamma\left[\varphi_{c l}\right]$ for a constant $\varphi_{c l}=\phi$. This amounts to an analysis of the effective potential, which is defined as [24, 25]

$$
\begin{aligned}
V(\phi) & =\left.\frac{\Gamma\left[\varphi_{c l}\right]}{\Omega}\right|_{\varphi_{c l}=\phi} \\
& =V_{0}(\phi)+\frac{\hbar}{2} \int \frac{d^{3} k}{(2 \pi)^{3}} \ln \left(k^{2}+M^{2}\right)-\left\langle\exp \left(-\frac{1}{\hbar} \int \frac{d^{3} k}{(2 \pi)^{3}} \mathcal{L}_{I}(\varphi, \phi)\right)\right\rangle,
\end{aligned}
$$

where $\Omega$ is the spacetime volume and $\mathcal{L}_{I}(\varphi, \phi)$ is the interaction part of the Lagrangian. The first term in Eq. (10) is the classical potential. It is given by the interaction term of the action without the kinetic contributions originated from the star product, that is,

$$
V^{(0)}(\phi)=\frac{1}{2} m^{2} \phi^{2}+\frac{g}{4 !} \phi^{4}+\frac{f}{6 !} \phi^{6} .
$$


The second term, containing the logarithm, is the contribution of all graphs with one closed loop. The third one is the sum of the higher-order loop corrections and is computed by taking the expectation value of

$$
T \exp \left(-\frac{1}{\hbar} \int \frac{d^{3} k}{(2 \pi)^{3}} \mathcal{L}_{I}(\varphi, \phi)\right)
$$

using conventional Feynman rules.

The relevant vertices for the two-loop calculation of the effective potential are depicted below:

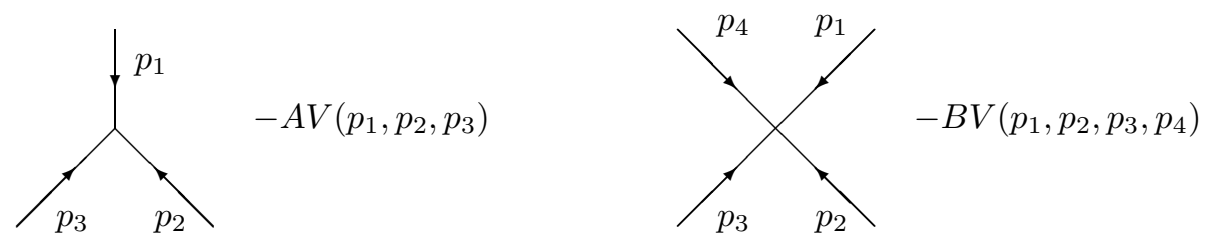

\section{EVALUATION OF THE EFFECTIVE POTENTIAL AT TWO-LOOP ORDER}

In this section we evaluate the effective potential for the $\varphi^{6}$ model up to two loops. Our calculations are rendered simpler by employing analytic regularization. The regularizing factor will always be assumed as finite. In what follows we shall work in units where $\hbar=1$.

The one-loop quantum contribution is evaluated using the trick

$$
\begin{aligned}
V^{(1)}(\phi) & =\frac{1}{2} \int \frac{d^{3} k}{(2 \pi)^{3}} \ln \left(k^{2}+M^{2}\right)=\frac{1}{2} \int d\left(M^{2}\right) \int \frac{d^{3} k}{(2 \pi)^{3}} \frac{1}{\left(k^{2}+M^{2}\right)^{(1+\epsilon)}} \\
& =\frac{1}{8 \pi^{3 / 2}} \frac{M^{(-2 \epsilon+3)}}{3-2 \epsilon} \frac{\Gamma\left(\epsilon-\frac{1}{2}\right)}{\Gamma(\epsilon+1)}=-\frac{M^{3}}{12 \pi}+O(\epsilon) .
\end{aligned}
$$

The two-loop correction is the sum of the double-bubble and sunset diagrams in their planar and nonplanar versions. The planar double-bubble is given by

$$
\begin{aligned}
\square & =D_{1 P}=-\frac{2}{3} \frac{B}{8} \int \frac{d^{3} k}{(2 \pi)^{3}} \frac{d^{3} p}{(2 \pi)^{3}} \frac{1}{\left(k^{2}+M^{2}\right)\left(p^{2}+M^{2}\right)} \\
& =-\frac{2}{3} \frac{B}{8} \frac{\pi^{3}}{(2 \pi)^{6}}\left(\int \frac{e^{-\alpha M^{2}}}{\alpha^{3 / 2-\epsilon}} d \alpha\right)^{2}=-\frac{B M^{2}}{192 \pi^{2}}+O(\epsilon) .
\end{aligned}
$$

The evaluation of the planar sunset can be done following the same procedure. It yields

$$
\begin{aligned}
\bigcirc & =D_{2 P}=\frac{1}{2} \frac{A^{2}}{12} \int \frac{d^{3} k}{(2 \pi)^{3}} \frac{d^{3} p}{(2 \pi)^{3}} \frac{1}{\left(k^{2}+M^{2}\right)\left(p^{2}+M^{2}\right)\left[(p+k)^{2}+M^{2}\right]} \\
& =\frac{1}{2} \frac{A^{2}}{12} \frac{\pi^{3}}{(2 \pi)^{6}} \int_{0}^{\infty} \alpha^{2} d \alpha \int_{0}^{1} d x \int_{0}^{1-x} d y \frac{e^{-\alpha M^{2}}}{\alpha[x(1-x)+y(1-y)-x y]^{1 / 2}} \\
& =\frac{A^{2}}{768 \pi^{2}} \Gamma(\epsilon)\left(\frac{M}{\bar{\mu}}\right)^{-2 \epsilon}=\frac{A^{2}}{768 \pi^{2} \epsilon}-\frac{A^{2}}{768 \pi^{2}} \ln \left(\frac{M^{2}}{\bar{\mu}^{2}} e^{\gamma}\right)+O(\epsilon),
\end{aligned}
$$

where $\bar{\mu}$ is an arbitrary constant with mass dimension and $\gamma$ is the Euler constant. In order that the effective potential do not be explicitly dependent on the polar terms, we can perform the redefinitions

$$
m_{R}^{2}=m^{2}-\frac{g^{2}}{384 \pi^{2} \epsilon} \quad, \quad g_{R}=g-\frac{f g}{96 \pi^{2} \epsilon} \quad, \quad f_{R}=f-\frac{5 f^{2}}{192 \pi^{2} \epsilon} .
$$


From now on, we shall assume the parameters of the model as defined by Eqs. (16) and omit the $R^{\prime} s$ from the notation. The expression for $D_{2 P}$ can therefore be written as

$$
D_{2 P}=-\frac{A^{2}}{768 \pi^{2}} \ln \left(\frac{M^{2}}{\mu^{2}}\right)
$$

where $\mu^{2}=\bar{\mu}^{2} e^{-\gamma}$.

The remaining contribution is given by the nonplanar versions of the diagrams (14) and (15), which are read as

$$
D_{1 N P}=-\frac{1}{3} \frac{B}{8} \int \frac{d^{3} k}{(2 \pi)^{3}} \frac{d^{3} p}{(2 \pi)^{3}} \frac{e^{i k_{\mu} \theta^{\mu \nu} p_{\nu}}}{\left(k^{2}+M^{2}\right)\left(p^{2}+M^{2}\right)}=-\frac{1}{3} \frac{B}{8} I_{1}
$$

and

$$
D_{2 N P}=\frac{1}{2} \frac{A^{2}}{12} \int \frac{d^{3} k}{(2 \pi)^{3}} \frac{d^{3} p}{(2 \pi)^{3}} \frac{e^{i k_{\mu} \theta^{\mu \nu} p_{\nu}}}{\left(k^{2}+M^{2}\right)\left(p^{2}+M^{2}\right)\left[(p+k)^{2}+M^{2}\right]}=\frac{1}{2} \frac{A^{2}}{12} I_{2} .
$$

By using the Feynman and Schwinger parametrizations, we can write $I_{1}$ as

$$
\begin{aligned}
I_{1} & =\int_{0}^{1} d w \int_{0}^{\infty} d \alpha \int \frac{d^{3} k d^{3} p}{(2 \pi)^{6}} \alpha e^{i k_{\mu} \theta^{\mu \nu} p_{\nu}} e^{-\alpha\left[\left(k^{2}-p^{2}\right) w+p^{2}+M^{2}\right]} \\
& =\int_{0}^{1} d w \int_{0}^{\infty} d \alpha \int \frac{d^{3} l d^{3} p}{(2 \pi)^{6}} \alpha e^{-\alpha w l^{2}} e^{-(1 / 4 \alpha w) \tilde{p}_{\mu} \tilde{p}^{\mu}} e^{-\alpha(1-w) p^{2}} e^{-\alpha M^{2}}
\end{aligned}
$$

where $\tilde{p}^{\mu}=\theta^{\mu \nu} p_{\nu}$ and $l^{\mu}=k^{\mu}-(i / 2 \alpha w) \theta^{\mu \nu} p_{\nu}$. In the frame defined by Eq. (2) $I_{1}$ is given by

$$
\begin{aligned}
I_{1} & =\int_{0}^{1} d w \int_{0}^{\infty} d \alpha \int \frac{d^{3} l d^{3} p}{(2 \pi)^{6}} \alpha e^{-\alpha w l^{2}} e^{-\left(\theta^{2} / 4 \alpha w\right)\left(p_{1}^{2}+p_{2}^{2}\right)} e^{-\alpha(1-w)\left(p_{0}^{2}+p_{1}^{2}+p_{2}^{2}\right)} e^{-\alpha M^{2}} \\
& =\frac{\pi^{3}}{(2 \pi)^{6}} \int_{0}^{\infty} d \alpha \int_{0}^{1} d w \frac{e^{-\alpha M^{2}}}{\left(\alpha^{2} w^{3 / 2}(1-w)^{3 / 2}+w^{1 / 2}(1-w)^{1 / 2} \frac{\theta^{2}}{4}\right)} \\
& =\frac{M^{2}}{32 \pi} \frac{\left[H_{0}\left(\theta M^{2}\right)-Y_{0}\left(\theta M^{2}\right)\right]}{\theta M^{2}}
\end{aligned}
$$

and, thus,

$$
D_{1 N P}=-\frac{B M^{2}}{768 \pi} \frac{\left[H_{0}\left(\theta M^{2}\right)-Y_{0}\left(\theta M^{2}\right)\right]}{\theta M^{2}},
$$

where $H_{0}(x)$ is the Struve function and $Y_{0}(x)$ is a Bessel function of the second kind. Using Feynman and Schwinger parametrizations, we can write $I_{2}$ as

$$
\begin{aligned}
I_{2} & =\int_{0}^{\infty} \alpha^{2} d \alpha \int_{0}^{1} d x \int_{0}^{1-x} d y \int \frac{d^{3} k d^{3} p}{(2 \pi)^{6}} e^{-\alpha(1-x) p^{2}} e^{-\alpha(1-y) k^{2}} e^{-2 \alpha(1-x-y) k_{\mu} p^{\mu}+i k_{\mu} \theta^{\mu \nu} p_{\nu}} e^{-\alpha M^{2}} \\
& =\int_{0}^{\infty} \alpha^{2} d \alpha \int_{0}^{1} d x \int_{0}^{1-x} d y \int \frac{d^{3} l d^{3} p}{(2 \pi)^{6}} e^{-\alpha(1-x) p^{2}} e^{-\alpha(1-y) l^{2}} e^{-[1 / 4 \alpha(1-y)]\left[-4 \alpha^{2}(1-x-y)^{2} p^{2}+\tilde{p}_{\mu} \tilde{p}^{\mu}\right]} e^{-\alpha M^{2}},
\end{aligned}
$$

where $l^{\mu}=k^{\mu}-[i / 2 \alpha(1-y)]\left[2 i(1-x-y) \alpha p^{\mu}+\theta^{\mu \nu} p_{\nu}\right]$. After a simplification, Eq. (23) is read as

$$
\begin{aligned}
I_{2}=\frac{\pi^{3}}{(2 \pi)^{6}} \int_{0}^{\infty} d \beta \int_{0}^{1} d x \int_{0}^{1-x} d y \frac{\beta e^{-\beta}}{[x(1-x)+} & y(1-y)-x y]^{1 / 2} \\
\times & \times \frac{1}{\left[[x(1-x)+y(1-y)-x y] \beta^{2}+\frac{\theta^{2} M^{4}}{4}\right]},
\end{aligned}
$$


where $\beta=\alpha M^{2}$. This is difficult to solve analytically. However, the contribution coming from this integral is finite and can be neglected if compared to the contribution coming from $I_{1}$. The finiteness of $I_{2}$ can be verified by considering the inequality

$$
\begin{aligned}
I_{2}\left(\theta M^{2}\right) & \leq \frac{\pi^{3}}{(2 \pi)^{6}} \int_{0}^{\infty} d \alpha \int_{0}^{1} d x \frac{\alpha e^{-\alpha M^{2}}}{[x(1-x)]^{1 / 2}} \frac{1}{\left[x(1-x) \alpha^{2}+\frac{\theta^{2}}{4}\right]} \\
& =-\frac{d}{d M^{2}} I_{1}=\frac{1}{32 \pi}\left[H_{1}\left(\theta M^{2}\right)-Y_{1}\left(\theta M^{2}\right)\right]-\frac{1}{16 \pi^{2}} .
\end{aligned}
$$

From Eq. (24) it is possible to determine the dependence of $I_{2}$ on $\theta$ as

$$
I_{2} \sim \ln \left(\theta^{2} M^{4}\right)+O(1)
$$

and from Eq. (21), we can write

$$
I_{1}=-\frac{M^{2}}{32 \pi^{2} \theta M^{2}} \ln \left(\frac{\theta^{2} M^{4}}{4} e^{2 \gamma}\right)+O(1) .
$$

Thus, when $\theta$ is sufficiently small, $I_{1}$ is clearly the dominant contribution, and $I_{2}$, as well as the one-loop correction, may be discarded. Among all the contributions other than the one of $D_{1 N P}$, we shall keep only the classical piece, since it is the dominant one for large $\phi$. Therefore, in the regime of small $\theta$, the effective potential is described by

$$
V(\phi)=\frac{m^{2}}{2} \phi^{2}+\frac{g}{4 !} \phi^{4}+\frac{f}{6 !} \phi^{6}+\frac{B M^{2}}{768 \pi} \frac{H_{0}\left(\theta M^{2}\right)-Y_{0}\left(\theta M^{2}\right)}{\theta M^{2}} .
$$

Notice that, when $\theta \rightarrow 0$, the nonplanar contribution behaves as $\ln \left(\Lambda_{\text {eff }}^{2} / M^{2}\right) \Lambda_{e f f}^{2} / M^{2}$, rather than as $\Lambda_{e f f}^{2} / M^{2}$. This may be attributed to the fact that $\theta^{0 i}=0$, which means that the effective cutoff is absent in the $p^{0}$ mode.

\section{ANALYSIS OF THE POTENTIAL}

\section{A. Tree-level Approximation}

Before considering the effective potential at two-loop order, it is interesting to initially make some remarks about the tree approximation [26]. In order that the potential have an absolute minimum, it is necessary that $f>0$. To perform the analysis of the vacuum, it is interesting to consider the parameter $f$ fixed, and vary $m^{2}$ and $g$. The possibilities for the variation of these parameters are (i) $m^{2} \geqslant 0$ and $g \geqslant 0$, (ii) $m^{2} \geqslant 0$ and $g<0$, (iii) $m^{2}<0$ and $g \geqslant 0$, and (iv) $m^{2}<0$ and $g<0$.

Figure 1 shows the shape of the effective potential at the tree level for all four possibilities in the thick solid lines of the graphs. In the first case [Fig.1(a)], there is no SSB and the potential presents a minimum at the origin. In the second, there is no SSB if $m^{2}>5 g^{2} / 8 f$ [Fig.1(b)], but it may occur if $m^{2}<5 g^{2} / 8 f$ [Fig.1(c)], being characterized by the presence of a local minimum at the origin and two global minima symmetrically disposed around it. Cases (iii) and (iv) (Fig.1d) present SSB with a maximum at the origin and two minima symmetrically disposed around it

\section{B. Two-loop potential}

A good evaluation of the modifications in the conditions for the SSB introduced by the noncommutative effects may be carried out by comparing the tree-level an two-loop corrected potentials in their noncommutative and commutative versions. The latter is obtained by summing up Eqs. (11), (13), and the planar diagrams (14) and (15) with their weights $2 / 3$ and $1 / 2$, respectively, redefined to be 1 . The resulting commutative two-loop potential is

$$
V(\phi)=\frac{m^{2}}{2} \phi^{2}+\frac{g}{4 !} \phi^{4}+\frac{f}{6 !} \phi^{6}-\frac{M^{3}}{12 \pi}+\frac{B M^{2}}{128 \pi^{2}}+\frac{A^{2}}{384 \pi^{2}} \ln \left(\frac{M^{2}}{\mu^{2}}\right)
$$



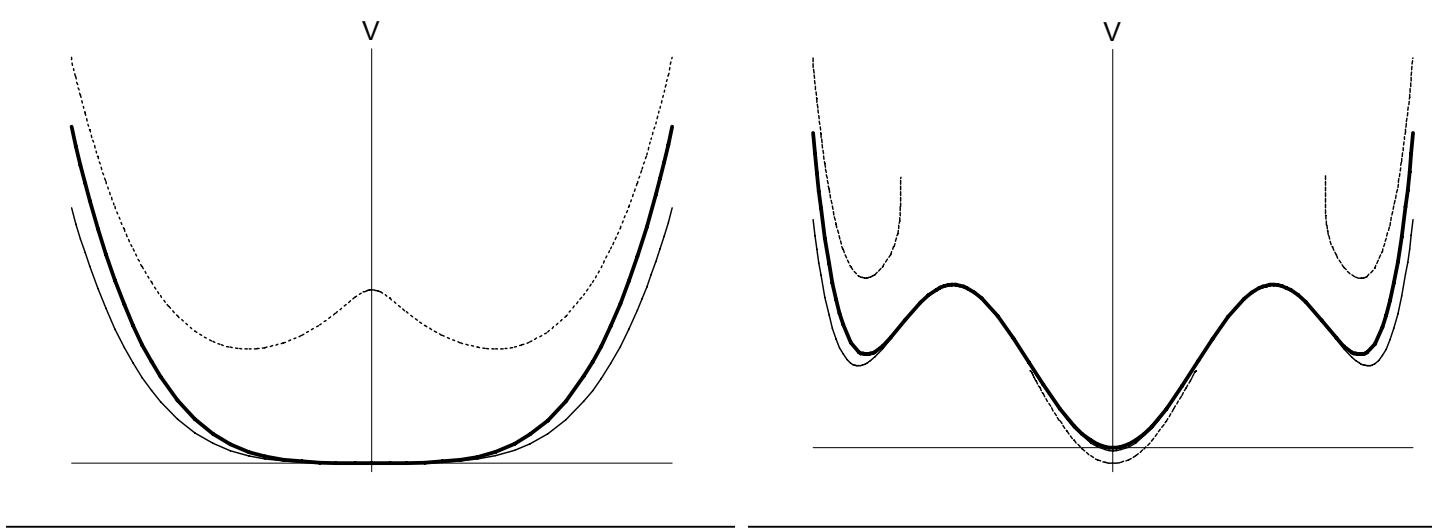

(a)

(b)
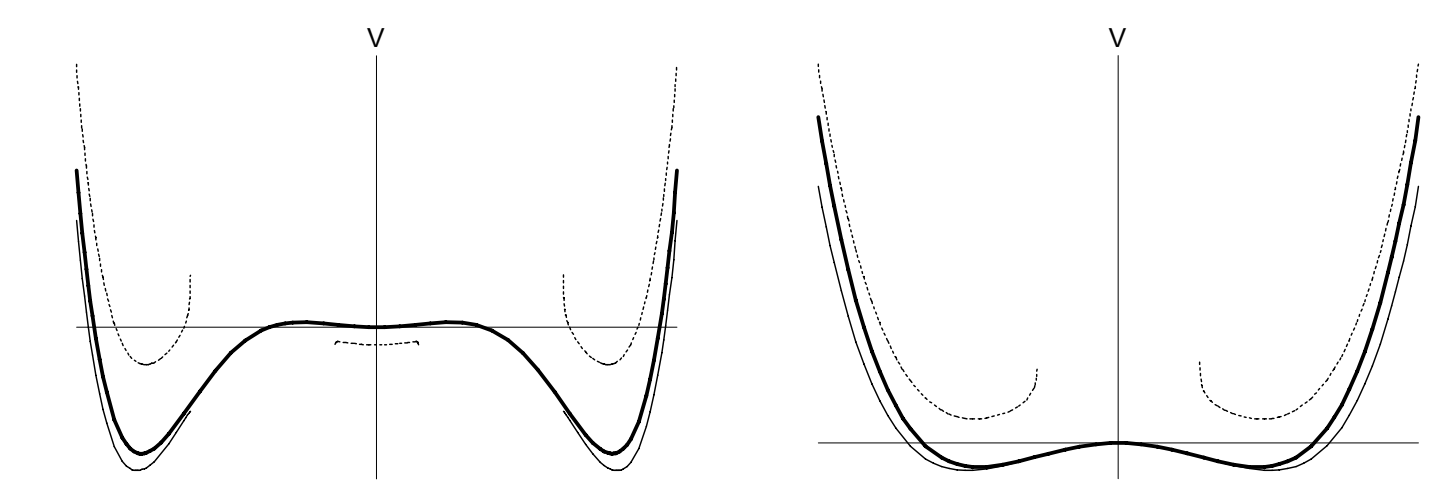

(c)

(d)

FIG. 1: A typical picture of the effective potential $V(\phi)$ at the tree level (thick solid lines) in contrast with its two-loop commutative (thin solid lines) and noncommutative (dotted lines) versions. (a) Case (i): $m^{2} \geqslant 0, g \geqslant 0$. (b) Case (ii): $m^{2} \geqslant 0, g<0\left(m^{2}>5 g^{2} / 8 f\right)$. (c) Case (iii): $m^{2} \geqslant 0, g<0\left(m^{2}<5 g^{2} / 8 f\right)$. (d) Cases (iii), (iv): $m^{2}<0, g \geqslant 0, g<0$.

In Fig. 1, it is possible to visualize the classical potential (thick solid lines), the two-loop corrected noncommutative potential (doted lines), and its two-loop corrected commutative counterpart (thin solid lines), for cases (i)-(iv).

In all cases, we see that the commutative two-loop corrections are manifest through small shifts in the minima, moving them downwards with respect to their positions at the tree level. The noncommutative twoloop corrections, on the other hand, tend to modify drastically the original shape of the potential. Notice that the dotted and thin solid lines are not continuous in cases (ii)-(iv). This may be attributed to the fact that the quantum corrections to the effective potential are not defined for the values of $\phi$ for which $M^{2}<0$. Thus, at the two-loop level, it is not possible to trace a complete picture of the potential with its minima in all cases. Since the corrections to the tree-level approximation may become big enough to displace the minima far from their original positions or alter their condition of minima, it is not possible to determine with certainty the position 


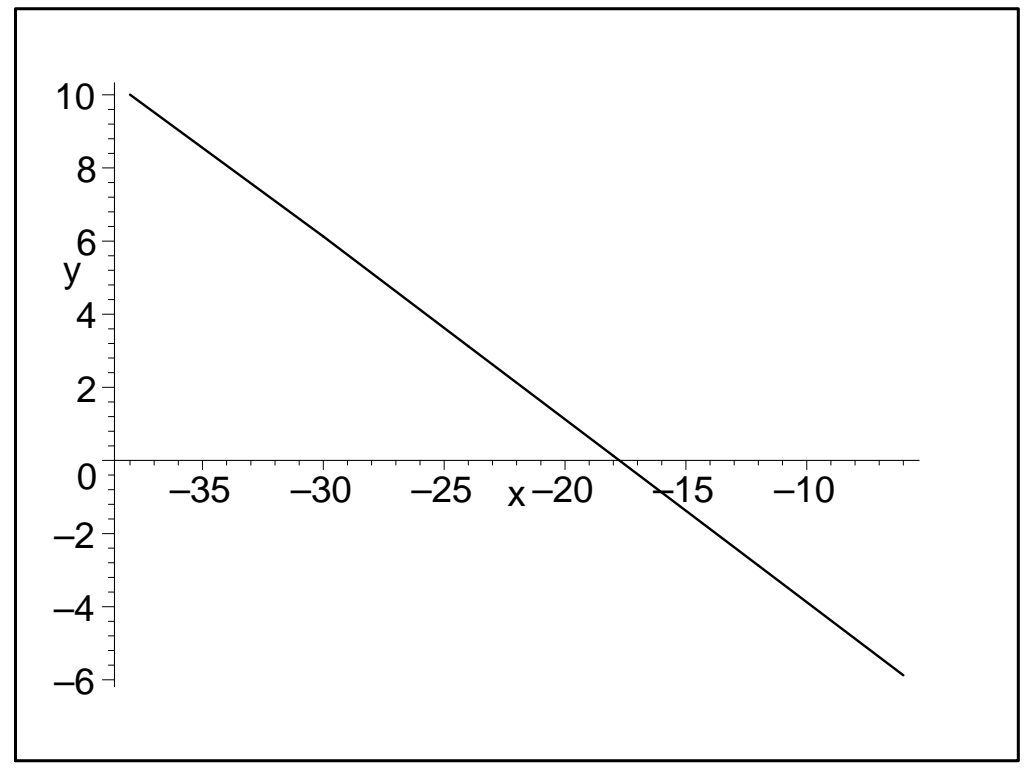

FIG. 2: $\mathbf{y}=\log _{10} m_{T}^{2}\left(\mathrm{GeV}^{2}\right)$, as a function of $x=\log _{10} \theta\left(\mathrm{GeV}^{-2}\right)$ for $f=4.4 \times 10^{-27}$, and $g=8.2 \times 10^{-8} \mathrm{GeV}$.

of the global minimum in cases (ii)-(iv). In connection with this, the most interesting case to be considered is depicted in Fig. (1a), where the quantum corrections are defined for all values of $\phi$, and a SSB is shown to be induced by noncommutativity effects. We shall, in what follows, restrict our attention to this case.

The conditions for SSB in case (i) can be determined by studying the concavity of the effective potential at the origin. Its second derivative at this point is given by

$$
\left.\frac{d^{2} V}{d \phi^{2}}\right|_{\phi=0}=m^{2}+\frac{m^{2}}{768 \pi \theta m^{2}}\left\{f\left[H_{0}\left(m^{2} \theta\right)-Y_{0}\left(m^{2} \theta\right)\right]-g^{2} \theta\left[H_{1}\left(m^{2} \theta\right)-Y_{1}\left(m^{2} \theta\right)-\frac{2}{\pi}\right]\right\} .
$$

Notice that $m^{2}$ and the first term between brackets are both positive. The second term containing $g^{2} \theta$, on the other hand, is negative and is the one which tends to modify the concavity of the potential. Given a fixed value for $\theta$, it is easy to see, thanks to the behavior of the functions $Y_{0}\left(m^{2} \theta\right) \sim \ln \left(m^{2} \theta\right)$ and $Y_{1}\left(m^{2} \theta\right) \sim-1 / m^{2} \theta$ near $m^{2} \theta=0$, that for sufficiently large $g$, and $m^{2}$ and $f$ sufficiently small, a reversal of concavity can occur.

Though in this work the formalism considered describes zero temperature, we can speculate and regard the indirect influence of finite temperature via physical parameters. A variation of temperature here could be regarded, for example, as equivalent to a change in the mass parameter $m$. By varying $m^{2}$, keeping $\theta$ fixed, it is easy to verify that the SSB generated is a second-order phase transition. ${ }^{1}$ To trace a picture of the dependence of the transition point $m_{T}^{2}$ on the noncommutativity scale, we can solve $d^{2} V /\left.d \phi^{2}\right|_{\phi=0}=0$ numerically for a wide range of values of $\theta$. Figure 2 depicts $y=\log _{10} m_{T}^{2}$ as a function of $x=\log _{10} \theta$ for given values of $f$ and $g$.

Since the values admissible for $\theta$ are small, an analytic approximation for $m_{T}^{2}$ can be obtained by expanding the right-hand side (RHS) of Eq. (29) in a series of $\theta$ :

$$
\left.\frac{d^{2} V}{d \phi^{2}}\right|_{\phi=0}=m^{2}-\frac{g^{2}}{384 \pi^{2} m^{2} \theta}-\frac{f}{384 \pi^{2} \theta} \ln \left(\frac{m^{2} \theta}{2} e^{\gamma}\right)+\frac{g^{2}}{384 \pi^{2}}+\frac{f}{384 \pi^{2}} m^{2}+O(\theta) .
$$

\footnotetext{
${ }^{1}$ We are considering the Landau definition that a transition is first order if the order parameter $\left(m^{2}\right)$ is discontinuous at the transition point and second order if it is continuous. Note that, for $n>2$, this differs from the Ehrenfest definition of an $n$th order transition as the one in which $\partial^{n} V / \partial\left(m^{2}\right)^{n}$ is the lowest discontinuous derivative.
} 
Discarding the last three terms on the RHS of Eq. (30), and solving $d^{2} V /\left.d \phi^{2}\right|_{\phi=0}=0$, we obtain

$$
m_{T}^{2}=\frac{g}{\sqrt{384 \pi^{2} \theta}}
$$

which reproduces the plot of Fig. 2 with great accuracy. In the ordinary commutative $\varphi^{6}$ model, $m_{T}^{2}=0$. Thus, as long as $g / \sqrt{\theta}$ is sufficiently big, the range of $m^{2}$ for which noncommutativity-induced SSB occurs, $0<m^{2}<m_{T}^{2}$, can be very large.

\section{Considerations of translational invariance}

Up to now, we have assumed translational symmetry as a hypothesis. As pointed out in [11], the SSB mechanism must involve the momentum modes where $\Gamma^{(2)}$ is smallest. The presence of a global minimum of $\Gamma^{(2)}\left(p_{0}^{2}, p_{n c}^{2}\right)$ away from $p_{\mu}=0$ prevents condensation of very-low-momentum modes and therefore breaks translational invariance. For $\theta$ sufficiently small, however, the minimum of $\Gamma^{(2)}$ here is always at $p_{\mu}=0$, in a similar way as occurs in [11]. The critical value $\theta_{c}$ at which the minimum starts to move away from $p_{\mu}=0$ is estimated in a self-consistent way, for case (i), as follows.

Substituting $\phi=0$ in the vertex factors and accounting for the possible crossings of the legs of the Feynman diagrams, it is possible to determine the shape of the leading contributions when $\Gamma^{(2)}$ is evaluated up to two-loop order $\mathrm{as}^{2}$

$$
\Gamma^{(2)}\left(p_{0}^{2}, p_{n c}^{2}\right) \sim p_{0}^{2}+p_{n c}^{2}+g \Lambda_{e f f}
$$

where $p_{n c}^{2}=p_{1}^{2}+p_{2}^{2}, \Lambda_{e f f}=1 / \sqrt{\theta^{2} p_{n c}^{2}+1 / \Lambda^{2}}$, and $\Lambda$ is a UV cutoff. We have neglected numerical coefficients and $p_{\mu}$-independent terms. In order that the extremum equation $\nabla \Gamma^{(2)}\left(p_{0}^{2}, p_{n c}^{2}\right)=0$ admit $p_{0}^{2}=p_{n c}^{2}=0$ as its unique solution, the following condition must be satisfied:

$$
\theta \Lambda^{2}<\left(\theta \Lambda^{2}\right)_{c} \sim 1 /(g / \Lambda)^{1 / 2}
$$

It is easy to check that the corresponding extremum is a minimum. We can fix $\Lambda$ and write Eq. (33) as $\theta<\theta_{c} \sim 1 /\left(g \Lambda^{3}\right)^{1 / 2}$ or fix $\theta$ and write Eq. (33) as $\Lambda<\Lambda_{c} \sim 1 /\left(g \theta^{2}\right)^{1 / 3}$. Let us fix some values for $\theta$ and compute the associated $\Lambda_{c}$ first. For a noncommutativity (NC) parameter around $\theta \sim 10^{-24} \mathrm{GeV}^{-2}$ (an experimental bound found by Anisimov et al. 13] from NCQED with $\Lambda=1 \mathrm{TeV}$ ), we have $\Lambda_{c} \sim 10^{18} \mathrm{GeV}$, near the Planck scale. On the other hand, if $\theta \sim 10^{-30} \mathrm{GeV}^{-2}$, which is the most stringent experimental bound proposed for this parameter (see Mocioiu et al. [12]), we have $\Lambda_{c} \sim 10^{22} \mathrm{GeV}$, which is beyond the Planck scale. For $\theta$ even smaller, the corresponding $\Lambda_{c}$ is yet larger. Since a reasonable value for $\Lambda$ is several times smallest than the Planck scale, the condition $\theta<\theta_{c} \sim 1 /\left(g \Lambda^{3}\right)^{1 / 2}$ can be easily satisfied. For $\Lambda=1 \mathrm{TeV}$, e.g., we would have $\theta_{c} \sim 10^{-1} \mathrm{GeV}^{-2} \gg 10^{-24} \mathrm{GeV}^{-2}$.

\section{DISCUSSION AND OUTLOOK}

In this work, we have carried out the study of aspects of SSB for the noncommutative $\varphi^{6}$ model. Our main goal was the investigation of the relevance of noncommutativity effects when $\theta$ is small. An analysis carried out at two-loop order in perturbation theory revealed that the noncommutative corrections to the effective potential are dominated by the nonplanar contributions. These are very different from the corresponding planar counterparts, which, except for weight factors, are functionally similar to the ordinary commutative ones. The bad behavior of the nonplanar diagrams in the $\theta \rightarrow 0$ limit can be interpreted as a consequence of

\footnotetext{
${ }^{2}$ We have discarded the two-loop corrections. For the values fixed for $f$ and $g$, it can be shown that their inclusion in the calculation does not significantly modify the estimate for $\theta_{c}$.
} 
the removal of the effective cutoff $\Lambda_{\text {eff }} \sim 1 / \sqrt{\theta}$, naturally introduced by noncommutative geometry. This is exactly what makes the study of the nonplanar contributions in the small- $\theta$ limit especially interesting, since they can drastically modify the shape of the effective potential. Indeed, this was the general conclusion of our qualitative analysis in all cases covered.

As the main result of our investigation, we found that, at the two-loop level, noncommutativity effects can induce a SSB for positive values of the mass parameter and the coupling constants. The conditions for this to occur were determined in a quantitative analysis, which provided an analytical expression for the square of the mass parameter, $m_{T}^{2}$, where the SSB takes place. For the values fixed for the coupling constants $g$ and $f$, the mass channel for which the noncommutativity-induced SSB occurs, $0<m^{2}<m_{T}^{2}$, was shown to comprise a reasonable range of values and to become large as long as $\theta$ gets smaller. Other choices for $g, f$ may yet expand the possibilities for the mass channel.

For the fixed values for $g$ and $f$, we showed that, for acceptable $\theta$ and reasonable values of $\Lambda$, translational symmetry can be assumed as a property of the vacuum state. If one considers $\Lambda$ as a parameter to vary, the corresponding $\theta_{c}(\Lambda)$ is a decreasing function. In the continuum limit (in the case it makes sense), $\theta_{c}=0$ and the translational invariant phase ceases to exist. The same occurs if one considers, e.g., the expression for $\theta_{c}$ presented for the $\varphi^{4}$ model in 11]: $\theta_{c} \sim 1 /\left(g \Lambda^{2}\right)$, where $g^{2}$ is the coupling constant. The existence of a translational invariant phase seems to be a common feature of the cutoff models, where, for $\theta$ sufficiently small, $\Gamma^{(2)}\left(p_{\mu}\right)$ admits its global minimum at $p_{\mu}=0$. In a previous work 20] it was argued that, for the noncommutative scalar field theories to be renormalizable, it would be necessary to relax the hypothesis of translational invariance. Perhaps translational invariance for small $\theta$ is therefore a property only of the cutoff models.

Beyond the natural interest that is motivating the investigation of noncommutativity from the theoretical point of view, knowledge of how it can modify the shape of the effective potential for scalar theories could be of great relevance, for example, in cosmology. The analysis of effective potentials plays a fundamental role in the study of inflationary universe models 27]. Since the $\theta^{i j}$ 's, if nonvanishing, should be very small, the results obtained in this work may be useful as clues for a possible application of noncommutativity as a mechanism for generating a symmetry breaking in the inflationary scenario. An example of how noncommutative field theory may be applied to inflationary cosmology may be found in [28].

\section{Acknowledgments}

The author is greatly indebted to José Helaÿel-Neto for discussions and all corrections on this manuscript. He also acknowledges Sebastião Alves Dias, Wolfgang Bietenholz, and Victor Rivelles for suggestions and a critical reading of earlier versions of the manuscript. This work was financially supported by CAPES.

[1] R. J. Szabo, Phys. Rep. 378, 207 (2003).

[2] M. R. Douglas and N. A. Nekrasov, Rev. Mod. Phys. 73, 977 (2002).

[3] N. Seiberg and E. Witten, J. High Energy Phys. 09, 032 (1999).

[4] A. Connes, M. R. Douglas and A. Schwarz, J. High Energy Phys. 02,003 (1998) 003; M R. Douglas and C. M. Hull, J. High Energy Phys. 02, 008 (1998).

[5] S. Doplicher, K. Fredenhagen and J. E. Roberts, Phys. Lett. B 331, 39 (1994); Comm. Math. Phys. 172, 187 (1995).

[6] A. P. Polychronakos, J. High Energy Phys. 04, 011 (2001);

L. Susskind, "The Quantum Hall Fluid and Noncommutative Chern-Simons Theory", hep-th/0101029

[7] J. Yeo, C. Rim and K. Moon, J. Phys. A 37, L39 (2004).

[8] S. Carroll, J. Harvey, V. A. Kostelecky, C. D. Lane and T. Okamoto, Phys. Rev. Lett. 87, 141601 (2001).

[9] S. Minwalla, M. Van Raamsdonk and N. Seiberg, J. High Energy Phys. 02, 020 (2000).

[10] B. A. Campbell and A. Kaminsky, Nucl. Phys. B 581, 240 (2000).

[11] S. S. Gubser and S. L.Sondhi, Nucl. Phys. B 605, 395 (2001).

[12] I. Mocioiu, M. Pospelov and R. Roiban, Phys. Lett. B 489, 390 (2000); 
G. Amelino-Camelia, L. Doplicher, S.-K. Nam and Y.-S. Seo, Phys. Rev. D 67085008 (2003);

I. Hinchliffe and N. Kersting, Int. J. Mod. Phys. A19, 179 (2004);

G. Amelino-Camelia, G. Mandanici and K. Yoshida, J. High Energy Phys. 01, 037 (2004).

[13] A. Anisimov, T. Banks, M. Dine and M. Graesser, Phys. Rev. D 65, 085032 (2002);

I. Hinchliffe and N. Kersting, Phys. Rev. D 64, 116007 (2001).

[14] Guang-Hong Chen and Yong-Shi Wu, Nucl. Phys. B 622, 189 (2002).

[15] G. Mandanici, Int. J. Mod. Phys. A19, 3541 (2004).

[16] W. H. Huang, Phys. Lett. B 496, 206 (2000).

[17] F. Ruiz Ruiz, Nucl. Phys. B 637,143 (2002).

[18] Y. Kiem, C. Kim and Y. Kim, Phys. Lett. B 507, 207 (2001).

[19] H. O. Girotti, M. Gomes, A. Yu. Petrov, V. O. Rivelles and A. J. da Silva, Phys. Rev. D 67, 125003 (2003).

[20] S. Sarkar, J. High Energy Phys. 02, 030 (2002).

[21] I. Chepelev and R. Roiban, J. High Energy Phys. 03, 001 (2001).

[22] W. Bietenholz, F. Hofheinz and J. Nishimura, Fortsch. Phys. 51, 745 (2003).

[23] S. Weinberg, The Quantum Theory of Fields, Cambridge University Press, 1996.

[24] R. Jackiw, Phys. Rev. D 9, 1686 (1974).

[25] M. Peskin and D. Schroeder, An Introduction to Quantum Field Theory, Perseus Books, Cambridge, Massashussets, 1995.

[26] K. Huang, Statistical Mechanics, John Wiley \& Sons, Second Edition, 1987.

[27] A. D. Linde, Rep. Prog. Phys. 42 (1979).

E. W. Kolb and M. S. Turner, The Early Universe, Perseus Publishing, 1994.

[28] C.-S. Chu, B. R. Greene and G. Shiu, Mod. Phys. Lett. A 16, 2231 (2001). 